\title{
High levels of leptin and non-high molecular weight-adiponectin in patients with colorectal cancer: Association with chemotherapy and common genetic polymorphisms
}

\author{
NIZAR M. MHAIDAT ${ }^{1}$, KAREM H. ALZOUBI ${ }^{1}$, MOHAMMED A. KUBAS $^{1}$, \\ MOHAMMED N. BANIHANI ${ }^{2}$, NASER HAMDAN ${ }^{1}$ and TAREQ M. AL-JABERI ${ }^{2}$ \\ ${ }^{1}$ Department of Clinical Pharmacy, Faculty of Pharmacy; ${ }^{2}$ Department of General Surgery and Urology, \\ Faculty of Medicine, Jordan University of Science and Technology, Irbid 22110, Jordan
}

Received June 20, 2020; Accepted October 27, 2020

DOI: $10.3892 /$ br.2020.1389

\begin{abstract}
Colorectal cancer (CRC) is the third most frequently diagnosed cancer worldwide. Leptin and adiponectin are hormones produced by adipose tissues, which exhibit opposing effects on tumor growth. Leptin promotes tumor development and metastasis, whereas adiponectin attenuates this. The aim of the present study was to assess the possible association between leptin and adiponectin [both high molecular weight (HMW) and non-HMW factions] levels with CRC, CRC response to chemotherapy, and to study the relationship between LEPR (rs6588147), ADIPO (rs266729), LEP (rs2167270), and ADIPO (rs822369) polymorphisms and CRC. A total of 32 blood samples collected from CRC patients were analyzed to identify the serum levels of leptin and adiponectin, and the presence of CRC related polymorphisms. A total of 25 healthy subjects were recruited in the control group. Serum levels of leptin and adiponectin were detected using ELISA whereas DNA from patients and controls was amplified and analyzed using PCR-restriction fragment length polymorphism assay. The results showed that the levels of leptin and non-HMW adiponectin were significantly higher in CRC patients compared with the controls $(\mathrm{P}<0.05)$. In addition, HMW adiponectin was significantly higher in patients receiving chemotherapy. The association between LEPR (rs6588147), ADIPO (rs266729), LEP (rs2167270) and ADIPO (rs822369)
\end{abstract}

Correspondence to: Professor Nizar M. Mhaidat, Department of Clinical Pharmacy, Faculty of Pharmacy, Jordan University of Science and Technology, Irbid 22110, Jordan

E-mail: nizarm@just.edu.jo

Abbreviations: CRC, colorectal cancer; BMI, body mass index; HMW, high-molecular weight; DM, Diabetes mellitus; HTN, hypertension

Key words: colorectal cancer, chemotherapy, leptin, adiponectin, patients, Jordan polymorphisms and $\mathrm{CRC}$ was not significant $(\mathrm{P}>0.05)$. In conclusion, higher leptin and non-HMW adiponectin levels may be associated with increased CRC. Chemotherapy may positively influence the levels of HMW adiponectin. No association between LEPR (rs6588147), ADIPO (rs266729), LEP (rs2167270) and ADIPO (rs822369) polymorphisms with CRC was found.

\section{Introduction}

Colorectal cancer (CRC) refers to a cancer that develops in the colon or in the rectum (1). CRC is characterized by an uncontrollable growth of cells in the terminal regions of the digestive system. CRC is the third most frequently diagnosed malignancy and the fourth leading cause of cancer-related death worldwide (2). In Jordan, the statistics show that CRC is the second most frequently diagnosed cancer and the second leading cause of cancer-related death in both males and females (3).

Obesity has been shown to contribute to an increased risk of cardiovascular disease and type 2 diabetes mellitus (DM) (4-6). Additionally, obesity was identified as a risk factor in the development of cancer and cancer-related death (7-9). According the American Cancer Society, excess body weight is hypothesized to be responsible for $\sim 8 \%$ of all types of cancer in the United States, as well as $~ 7 \%$ of all cancer deaths (10). Overweight teenagers with colon cancer have a $2 \mathrm{x}$ risk of mortality in adulthood (11).

Adipose tissue dysfunction is one hypothesis that may link obesity and CRC due to secretion of several hormones called adipokines, the most important of which are leptin and adiponectin (12). Leptin, the product of the $o b$ gene, is a $16 \mathrm{kDa}$ peptide cytokine, which is produced exclusively by adipose tissues. Leptin primarily regulates food intake and energy expenditure (13), and its concentration is positively correlated with the body mass index (BMI), and is thus higher in obese individuals (14). Several experimental studies have shown that leptin has a mitogenic, antiapoptotic and tumorigenic effect on different cancer cell lines, including colorectal, lung, breast, thyroid, pancreatic and uterine cancer cells (15-17). In addition, in CRC tissues, it was found that leptin and its receptor 
were significantly upregulated compared with normal tissues and its upregulation was associated with a more advanced tumor phenotype $(18,19)$.

Adiponectin is a protein with a molecular weight of $30 \mathrm{kDa}$ which is derived primarily from adipocytes (20). Its anti-atherogenic and anti-inflammatory properties, as well as its ability to sensitize cells to insulin are considered the most important functions of adiponectin (21). Adiponectin circulates in the blood in different forms including as a trimer, hexamer and high-molecular weight (HMW) complexes. These forms were found to have different biological activities (22). For example, HMW adiponectin affects insulin sensitivity, whereas the anti-inflammatory activity of the non-HMW is its prominent effects (23).

An inverse relationship between obesity, insulin resistance, type $2 \mathrm{DM}$ and the serum levels of adiponectin has been identified (24). Obesity and DM are associated with a higher incidence of CRC (25). Studies have found that lower levels of total adiponectin are associated with an increased risk of colorectal cancer $(26,27)$. Conversely, other studies have found higher levels of total adiponectin in patients with CRC compared with the control $(28,29)$. Thus, additional studies are required to highlight the association between adiponectin levels and CRC.

The aims the present study were to determine the relationship between leptin and adiponectin levels on the risk of CRC and response to chemotherapy. Furthermore, the association between LEPR (rs6588147), ADIPO (rs266729), LEP (rs2167270) and ADIPO (rs822369) polymorphisms and CRC occurrence were investigated.

\section{Materials and methods}

Ethical approval. The present study was approved by the Institutional Review Board of Jordan University of Science and Technology (approval no. 80/2012). Written informed consent was obtained from all study participants.

Study population and blood collection. Blood samples were collected at King Abdullah University Hospital, including 32 CRC patients who were histopathologically diagnosed with colorectal adenocarcinoma; 13 of these were female and 19 were male. The median age was 52 years old (age range, 21-75 years). Additionally, 25 healthy volunteers were recruited of which, 5 were female and 20 were male. The median age was 30 years old (age range, 18-59 years). Samples of control individuals were chosen from fully healthy subjects with a similar BMI. Exclusion criteria were: i) A previous gastrointestinal tract surgery; ii) inflammatory bowel disease (ulcerative colitis or Crohn's disease); iii) patients with a cancer in a different organ; iv) acute or chronic infections; v) familial adenomatous polyposis; vi) autoimmune disease; vii) rheumatoid arthritis; viii) malnutrition; ix) other primary cachectic states (such as congestive pulmonary disease or cirrhosis); or $\mathrm{x}$ ) lack of consent to participate in the study. Data regarding the histopathological diagnosis, staging of CRC patients, whether patients started on chemotherapy and the number of cycles given, were retrieved from the medical records. The CRC patients were classified according to
Table I. Primer sequences.

Genotype

Primer, 5'-3'

$\begin{array}{ll}\text { LEP R (rs6588147) } & \\ \text { Forward } & \text { GCATATGCCTGTGTGCAGTT } \\ \text { Reverse } & \text { GCAGTTCTGGATCAGTGCAA } \\ \text { ADIPO (rs266729) } & \\ \text { Forward } & \text { CATCAGAATGTGTGGCTTGC } \\ \text { Reverse } & \text { AGAAGCAGCCTGGAGAACTG } \\ \text { LEP (rs2167270) } & \\ \text { Forward } & \text { GATCGGGCCGCTATAAGAG } \\ \text { Reverse } & \text { GCATCCCTCCTGACTCAGTT } \\ \text { ADIPO (rs822369) } & \\ \text { Forward } & \text { CTGAAGTTTGGGGACAAACAG } \\ \text { Reverse } & \text { CTTTTCTGCTGGGAAACAGG }\end{array}$

the Tumor-Node-Metastasis staging system and Dukes's classification $(30,31)$.

Blood samples, which were collected from all patients and controls in the morning, were split equally in to two different tubes (plain and EDTA tubes). The serum blood obtained from the plain tubes was allowed to coagulate at room temperature for $30 \mathrm{~min}$, and then centrifuged at $1,968 \mathrm{x} \mathrm{g}$ for $10 \mathrm{~min}$ at $4^{\circ} \mathrm{C}$. The supernatant was separated from the samples and transferred into PCR tubes $(100 \mu \mathrm{l})$, and stored at $-20^{\circ} \mathrm{C}$ until required. The EDTA blood samples were stored at $-8^{\circ} \mathrm{C}$ until required.

Biochemical analysis. Serum concentrations of leptin, and adiponectin (total and HMW) were measured using ALPCO Immunoassay kits using ELISA. Leptin levels were determined according to the manufacturer's protocol (Leptin ELISA kit; cat. no. 11-LEPHU-E01; sensitivity, $0.5 \mathrm{ng} / \mathrm{ml}$; ALPCO). Plasma concentrations of adiponectin (total and HMW) were analyzed according to manufacturer's protocol [Adiponectin (Multimeric) ELISA kit; cat. no. 47-ADPHU-E01; sensitivity, $0.019 \mathrm{ng} / \mathrm{ml}$, ALPCO].

DNA extraction and genotyping. The DNA was extracted from the EDTA blood samples of CRC patients and control participants according to the manufacturer's protocol (Wizard Genomic DNA Purification kit; Promega corporation). The LEPR (rs6588147), ADIPO (rs266729), LEP (rs2167270) and ADIPO (rs822369) polymorphisms were analyzed by PCR-restriction fragment length polymorphism assay. The sequences of the primers were designed according to their sequence gene and purchased from Integrated DNA Technologies, Inc. (Table I).

Amplification of the 194 and 163 bp PCR products for LEPR (rs6588147) and ADIPO (rs266729), respectively, was performed using the following thermocycling conditions: Initial denaturation at $95^{\circ} \mathrm{C}$ for $5 \mathrm{~min}$; followed by 35 cycles of $95^{\circ} \mathrm{C}, 54^{\circ} \mathrm{C}$ and $72^{\circ} \mathrm{C}$ for $90 \mathrm{sec}$ each; and a final extension at $72^{\circ} \mathrm{C}$ for $7 \mathrm{~min}$. Amplification of the 176 and $214 \mathrm{bp}$ PCR products for LEP (rs2167270) and ADIPO (rs822369), respectively, was performed as follows: Initial denaturation 
Table II. Demographic and biomarkers in controls vs. CRC patients.

\begin{tabular}{lccc}
\hline Characteristic & Value & Controls & CRC patients \\
\hline Sex, $\mathrm{n}(\%)$ & & & P-value \\
Male & $39(68.4)$ & $20(35.1)$ & $19(33.3)$ \\
Female & $18(31.6)$ & $5(8.8)$ & $13(22.8)$ \\
Age, years & $52.85 \pm 11.51$ & $52.80 \pm 11.46$ & $54.10 \pm 13.58$ \\
Body mass index, $\mathrm{kg} / \mathrm{m}^{2}$ & $25.90 \pm 4.28$ & $24.31 \pm 3.26$ & $27.26 \pm 4.62$ \\
Leptin, ng/ml & $10.17 \pm 8.64$ & $7.13 \pm 7.59$ & $12.35 \pm 8.79$ \\
Total adiponectin, $\mathrm{ng} / \mathrm{ml}$ & $3,459.84 \pm 1,911.42$ & $3,051.21 \pm 1,162.42$ & $3,753.54 \pm 2,278.68$ \\
HMW adiponectin, $\mathrm{ng} / \mathrm{ml}$ & $924.81 \pm 1,326.86$ & $1,175.84 \pm 1,206.90$ & $744.39 \pm 1,397.40$ \\
Non-HMW adiponectin, ng/ml & $0.244 \pm .264$ & $1,875.36 \pm .1,079.18$ & $3,009.15 \pm 1,856.58$ \\
\hline
\end{tabular}

${ }^{\mathrm{a}} \mathrm{P}<0.05,{ }^{\mathrm{b}} \mathrm{P}<0.01$. Data are presented as the mean \pm standard deviation unless otherwise is indicated. HMW, high molecular weight.

Table III. Association between biomarkers and characteristics of patients.

\begin{tabular}{lcccc}
\hline Characteristic (n) & Leptin & Total adiponectin & HMW adiponectin & Non-HMW adiponectin \\
\hline Sex & & & & \\
$\quad$ Male (19) & $10.91 \pm 8.95$ & $3,714.77 \pm 2,615.12$ & $674.55 \pm 1,553.16$ & $3,040.22 \pm 2,206.16$ \\
$\quad$ Female (13) & $14.46 \pm 8.46$ & $3,810.20 \pm 1,774.69$ & $846.46 \pm 1,186.19$ & $2,963.74 \pm 1,264.91$ \\
Age, years & & & & \\
$\quad<40(4)$ & $15.48 \pm 8.69$ & $5,244.59 \pm 1,310.39$ & $1,859.1 \pm 1,671.46^{\mathrm{a}}$ & $3,385.49 \pm 537.07$ \\
$40-60(14)$ & $12.36 \pm 7.27$ & $2,706.97 \pm 1,429.06$ & $310.08 \pm 2,90.80$ & $2,396.88 \pm 1,327.58$ \\
$>60(11)$ & $13.69 \pm 10.78$ & $4,126.87 \pm 2,803.74$ & $382.49 \pm 565.81$ & $3,744.37 \pm 2,592.83$ \\
Body mass index, $\mathrm{kg} / \mathrm{m}^{2 \mathrm{~b}}$ & & & & \\
$<24.99(8)$ & $9.47 \pm 9.01$ & $4,348.27 \pm 3,561.70$ & $1,038.84 \pm 1,359.09$ & $3,309.42 \pm 3,163.05$ \\
$25-29.99(13)$ & $12.34 \pm 7.82$ & $3,274.84 \pm 1,466.06$ & $362.27 \pm 390.93$ & $2,912.56 \pm 1,360.60$ \\
$>30(8)$ & $19.16 \pm 8.51$ & $3,277.26 \pm 1,529.37$ & $390.93 \pm 515.41$ & $2,993.47 \pm 1,175.47$
\end{tabular}

${ }^{\mathrm{a}} \mathrm{P}<0.05$. $^{\mathrm{b}} \mathrm{Data}$ missing for 3 patients. Data are presented as the mean \pm standard deviation. HMW, high molecular weight.

at $95^{\circ} \mathrm{C}$ for $5 \mathrm{~min}$; followed by 35 cycles of $95^{\circ} \mathrm{C}, 53^{\circ} \mathrm{C}$ and $72^{\circ} \mathrm{C}$ for $90 \mathrm{sec}$ each; and a final extension at $72^{\circ} \mathrm{C}$ for $7 \mathrm{~min}$. Amplification was performed on a Kyratec SuperCycler SC200 and the corresponding included software (software version 3.0.1, Hardware Version 3; Kyratec).

Statistical analysis. Data are presented as the mean \pm standard deviation. SPSS version 25 (IBM, Corp.) was used for statistical analysis. A $\chi^{2}$ test was used to assess differences amongst categorical groups, whereas a Student's t-test (two-tailed) was used to compare differences between continuous variables. A one-way ANOVA followed by a Bonferroni post-hoc test was used to compare differences between $>2$ groups. $\mathrm{P}<0.05$ was considered to indicate a statistically significant difference.

\section{Results}

Demographics and biomarkers. There was no significant difference in the demographics between the patients and control groups. Plasma concentrations of leptin were significantly higher in the CRC group compared with the control group $(\mathrm{P}<0.05)$. Compared with the controls, non-HMW adiponectin levels were significantly higher in CRC patients. However, serum concentrations of both total and HMW adiponectin were not significantly different between the two groups (Table II).

Table III shows that the sex, age and BMI variations amongst CRC patients were not significantly associated with leptin, total adiponectin, HMW adiponectin or non-HMW adiponectin levels. Nevertheless, in patients aged $<40$ years, HMW adiponectin was significantly higher compared with patients $>40$ years.

Comorbidities of CRC patients. The majority of the patients enrolled in the present study were free of any chronic disease $(56.3 \%)$, whereas 4 patients $(12.5 \%)$ had DM either alone (1 patient, $3.1 \%$ ) or with another comorbidity, such as hypertension (HTN), chronic kidney disease (CKD) or hyperlipidemia (3 patients, 9.4\%). HTN was observed in (7 patients, $21.9 \%$ ), of whom 5 patients $(15.6 \%)$ had HTN alone, whereas 2 patients $(6.3 \%)$ had HTN and ischemic heart disease. The data of the 3 patients with comorbidities was not recorded. 
Table IV. Association between biomarkers and duration of diagnosis, stages, chemotherapy and the number of cycles of chemotherapy in patients.

\begin{tabular}{lcccc}
\hline Parameter & Leptin & Total adiponectin & HMW adiponectin & Non-HMW adiponectin \\
\hline Duration of diagnosis, years (n) & & & & \\
0-1 (7) & $13.05 \pm 9.25$ & $2,455.18 \pm 888.06$ & $269.66 \pm 155.98$ & $2,185.52 \pm 890.47$ \\
$1-2(14)$ & $12.61 \pm 8.51$ & $3,456.60 \pm 1,779.24$ & $608.90 \pm 1,128.98$ & $2,847.69 \pm 1,365.38$ \\
2-5 (7) & $12.86 \pm 8.58$ & $5,050.55 \pm 3,296.51$ & $776.62 \pm 683.90$ & $4,273.93 \pm 3,093.65$ \\
$>5$ (1) & 27.6 & $3,338.96$ & 136.36 & $3,202.59$ \\
Disease stage (n) & & & & $2,669.32 \pm 1,236.92$ \\
Stage I (3) & $12.76 \pm 6.57$ & $2,706.25 \pm 1,206.27$ & $59.88 \pm 21.63$ & $2,845.48 \pm 1,674.51$ \\
Stage II (5) & $17.94 \pm 9.17$ & $3,252.59 \pm 1,795.73$ & $407.11 \pm 637.04$ & $2,365.70 \pm 902.13$ \\
Stage III (9) & $10.04 \pm 7.47$ & $3,090.12 \pm 1,697.61$ & $724.42 \pm 1,309.33$ & \\
Chemotherapy (n) & & & & $3,103.01 \pm 1,111.27$ \\
Yes (13) & $16.07 \pm 8.72$ & $4,024.19 \pm 1,613.17$ & $921.17 \pm 1,174.39$ & $2,996.71 \pm 2,419.77$ \\
No (16) & $11.03 \pm 8.22$ & $3,247.31 \pm 2,579.27$ & $250.60 \pm 241.65$ & $2,113.20 \pm 97.41$ \\
Treatment cycles (n) & & & & $5,468.86 \pm 3,253.69$ \\
1-3 (3) & $26.40 \pm 3.79$ & $2,287.80 \pm 192.22$ & $174.61 \pm 128.66$ & $2,910.86 \pm 8,36.91$ \\
4-6 (5) & $7.34 \pm 5.20$ & $6,857.99 \pm 2,983.28$ & $1,389.12 \pm 1,630.81$ & $916.47 \pm 684.18$ \\
$\geq 7$ (5) & $17.86 \pm 6.36$ & $3,642.25 \pm 1,367.20$ & & \\
\hline
\end{tabular}

${ }^{\mathrm{a}} \mathrm{P}<0.05$. HMW, high molecular weight. ${ }^{\mathrm{b}}$ The majority of patients received FOLFOX4 chemotherapy regimen except one patient who received FOLFOX4 plus bevacizumab. Data are presented as the mean \pm standard deviation. FOLFOX4, Oxaliplatin, Leucovorin, 5-Fluorouracil.

Table V. Incidence of polymorphisms in control vs. patients.

\begin{tabular}{lccc}
\hline Genotype & Total samples, $(\%)$ & Controls, $n(\%)$ & CRC patients, n $(\%)$ \\
\hline LEPR (rs6588147) & & & $14(25.9)$ \\
AA & $29(53.7)$ & $6(11.1)$ & $14(25.9)$ \\
AG & $20(37.0)$ & $3(5.6)$ & $2(3.7)$ \\
GG & $5(9.3)$ & & $19(35.2)$ \\
ADIPO (rs266729) & & $11(20.4)$ & $9(16.7)$ \\
CC & $30(55.6)$ & $10(18.5)$ & $3(5.6)$ \\
CG & $19(35.2)$ & $2(3.7)$ & $13(24.1)$ \\
GG & $5(9.3)$ & $8(14.8)$ & $12(22.2)$ \\
LEP (rs2167270) & $21(38.9)$ & $11(20.4)$ & $6(11.1)$ \\
GG & $23(42.6)$ & $4(7.4)$ & 0.795 \\
GA & $10(18.5)$ & $7(13.0)$ & $3(5.6)$ \\
AA & $10(18.5)$ & $4(7.4)$ & $7(13.0)$ \\
ADIPO (rs822396) & $11(20.4)$ & $12(22.2)$ & $21(38.9)$ \\
AA & $33(61.1)$ & & 0.152 \\
AG & & & \\
GG & & & \\
\hline
\end{tabular}

CRC, colorectal cancer.

Association between biomarkers and diagnosis, duration, disease stage and chemotherapy cycles. The CRC patients who were on chemotherapy had significantly higher levels of HMW adiponectin compared with those who did not take chemotherapy (Table IV). For the duration of diagnosis, disease stage, chemotherapy cycles and biomarkers were not significant different between the control and CRC patients.

Incidence of polymorphisms. There was no significant difference between CRC patients and control subjects in the frequency of LEPR (rs6588147), ADIPO (rs266729), LEP 
(rs2167270) and ADIPO (rs822369) polymorphisms ( $\mathrm{P}>0.05$; Table V).

\section{Discussion}

The present study showed that leptin and non-HMW adiponectin levels were significantly higher in CRC patients compared with the controls. In addition, HMW adiponectin levels were significantly higher in patients who received chemotherapy compared with those who did not, whereas leptin levels were not significantly different between patients who received chemotherapy and those who did not. In addition, there was no association between LEPR (rs6588147), ADIPO (rs266729), LEP (rs2167270) and ADIPO (rs822369) polymorphisms and CRC.

The association between leptin levels and CRC remains controversial. In a prospective study, leptin concentration was found to be similar in CRC patients compared with the controls (32), whereas other studies reported a significant decrease in leptin serum levels in CRC patients $(33,34)$. In agreement with the present study, studies have shown that leptin is significantly associated with CRC occurrence $(35,36)$, and this may due to the established tumorigenic, mitogenic and antiapoptotic effects of leptin $(15,16)$. However, sex differences between control (5 females, 20 males) and CRC patients (13 females, 19 males) may influence leptin levels. Previous studies have shown females have 2-3 times higher levels of leptin than males regardless of BMI; higher fat mass may explain the higher levels of leptin in circulation in females compared with males (37-39). Therefore, in the present study, it is possible that sex variations may have contributed to the higher levels of leptin shown amongst CRC patients.

The results of the present study showed higher levels of non-HMW adiponectin in CRC patients compared with the control group, which may be closely associated with CRC occurrence. In contrast, a meta-analysis indicated that a low circulating non-HMW fraction may be considered as a risk factor for CRC (40). Increased levels of non-HMW adiponectin in CRC patients shown in the present study may be associated with the involvement of non-HMW adiponectin in the regulation of inflammatory processes, which is considered a key factor in cancer progression and metastasis in various types of cancer including CRC $(23,41)$. However, the exact mechanism that may explain the higher levels of non-HMW adiponectin in CRC patients is unknown, and further studies are required to this end.

In the present study, CRC patients receiving chemotherapy had significantly higher HMW adiponectin levels compared with those who did not receive chemotherapy. Thus, it is likely that chemotherapy may have resulted in such an elevation. However, no significant difference in leptin levels was detected in patients who received chemotherapy compared with those who did not. A previous study has reported that adiponectin levels are increased after receiving chemotherapy in patients with CRC who exhibited a partial response (42) and in patients with lung cancer (43). These results may indicate that adiponectin levels are sensitive to chemotherapy (44). Therefore, further studies are required to evaluate the potential value of adiponectin levels as predictive or prognostic markers for the chemotherapy response (45); specifically, HMW adiponectin.
The results of the present study showed there was no association between LEPR (rs6588147), ADIPO (rs266729), LEP (rs2167270) and ADIPO (rs822369) polymorphisms and CRC. In agreement, Carvajal-Carmona et al (46) did not find an association between the ADIPO (rs266729) polymorphisms and CRC. However, previous studies have reported an association between these variants and CRC, particularly LEP (rs2167270) and ADIPO (rs266729) (47-49). The difference in the ethnic groups and the smaller number of samples may explain the differences in results.

The present study was performed on CRC patients from a large health center; however, it has some limitations. First, a small number of patients were enrolled. Secondly, due to the limited number of participants and less willingness of female participants to be involved in the study, we could not match for sex between control and patient group. Thirdly, due to sample size limitations, the results of this study are based only on univariate analyses. Finally, the effect of leptin on migration and invasion of CRC was not investigated. Thus, a future study that is more comprehensive and addressing whether leptin promotes migration and invasion in CRC patients is required.

In conclusion, the present study showed that higher plasma levels of leptin and non-HMW adiponectin may be associated with an increased risk of CRC, whereas HMW adiponectin levels may increase with chemotherapeutic regimens. The polymorphisms of LEPR (rs6588147), ADIPO (rs266729), LEP (rs2167270) and ADIPO (rs822369) were not associated with CRC.

\section{Acknowledgements}

Not applicable.

\section{Funding}

This study was supported by funding from the Deanship of Research at Jordan University of Science and Technology (grant no. 80/2012).

\section{Availability of data and materials}

The datasets used and/or analyzed during the current study are available from the corresponding author on reasonable request.

\section{Authors' contributions}

NMM designed the study, assisted in collection of patient samples, contributed to the analysis and interpretation of the data, and assisted in manuscript writing. KHA was involved in the study conception and design, sample analysis and interpretation, and assisted in writing the manuscript. MAK participated in study design, collection of patient data, performed the molecular analysis of the samples, and assisted in manuscript writing. MNB was involved in the study design, subject recruitment and data interpretation. NH participated in study design, assisted in molecular analysis of the samples, and in manuscript writing. TMA was involved in study design, participated in collection of patient samples, interpretation of results and manuscript writing. All authors have read and approved the final manuscript. 


\section{Ethics approval and consent to participate}

The present study was approved by the Institutional Review Board of Jordan University of Science and Technology (approval no. 80/2012). Written informed consent was obtained from all study participants.

\section{Patient consent for publication}

Not applicable.

\section{Competing interests}

The authors declare that they have no competing interests.

\section{References}

1. American Cancer Society: About colorectal cancer-overview and types. American Cancer Society, Inc., Atlanta, GA, 2018 https://www.cancer.org/cancer/colon-rectal-cancer/about.html.

2. Arnold M, Sierra MS, Laversanne M, Soerjomataram I, Jemal A and Bray F: Global patterns and trends in colorectal cancer incidence and mortality. Gut 66: 683-691, 2017.

3. Sharkas GF, Arqoub KH, Khader YS, Tarawneh MR, Nimri OF, Al-Zaghal MJ and Subih HS: Colorectal cancer in Jordan: Survival rate and its related factors. J Oncol 2017: 3180762, 2017

4. Calle EE, Thun MJ, Petrelli JM, Rodriguez C and Heath CW Jr: Body-mass index and mortality in a prospective cohort of U.S. adults. N Engl J Med 341: 1097-1105, 1999.

5. Hubert HB, Feinleib M, McNamara PM and Castelli WP: Obesity as an independent risk factor for cardiovascular disease: A 26-year follow-up of participants in the Framingham heart study. Circulation 67: 968-77, 1983.

6. Mokdad AH, Ford ES, Bowman BA, Nelson DE, Engelgau MM, Vinicor F and Marks JS: Diabetes trends in the U.S.: 1990-1998 Diabetes Care 23; 1278-1283, 2000.

7. World Cancer Research Fund, American Institute for Cancer Research (AICR): Food, nutrition, physical activity, and the prevention of cancer: A global perspective. AICR, Washington, DC, 2007.

8. Ungefroren H, Gieseler F, Fliedner S and Lehnert H: Obesity and cancer. Horm Mol Biol Clin Investig 21: 5-15, 2015.

9. Colditz GA, Wolin KY and Gehlert S: Applying what we know to accelerate cancer prevention. Sci Transl Med 4: 127rv4, 2012.

10. American Cancer Society: Body weight and cancer risk. American Cancer Society, Inc., Atlanta, GA, 2015. https://www. cancer.org/cancer/cancer-causes/diet-physical-activity/bodyweight-and-cancer-risk/effects.html.

11. Bjørge T, Engeland A, Tverdal A and Smith GD: Body mass index in adolescence in relation to cause-specific mortality: A follow-up of 230,000 Norwegian adolescents. Am J Epidemiol 168: 30-37, 2008.

12. van Kruijsdijk RC, van der Wall E and Visseren FL: Obesity and cancer: The role of dysfunctional adipose tissue. Cancer Epidemiol Biomarkers Prev 18: 2569-2578, 2009.

13. Zhang Y, Proenca R, Maffei M, Barone M, Leopold L and Friedman JM: Positional cloning of the mouse obese gene and its human homologue. Nature 372: 425-432, 1994.

14. Münzberg H and Myers MG Jr: Molecular and anatomical determinants of central leptin resistance. Nat Neurosci 8: 566-570, 2005.

15. Hardwick JCH, Van Den Brink GR, Offerhaus GJ, Van Deventer SJH and Peppelenbosch MP: Leptin is a growth factor for colonic epithelial cells. Gastroenterology, 2001.

16. Aparicio T, Kotelevets L, Tsocas A, Laigneau JP, Sobhani I, Chastre E and Lehy T: Leptin stimulates the proliferation of human colon cancer cells in vitro but does not promote the growth of colon cancer xenografts in nude mice or intestinal tumorigenesis in Apc(Min/+) mice. Gut 54: 1136-1145, 2005.

17. Samad N and Rao T: Role of leptin in cancer: A systematic review. Biomed J Sci Tech Res 18: 13226-13235, 2019.

18. Paik SS, Jang SM, Jang KS, Lee KH, Choi D and Jang SJ: Leptin expression correlates with favorable clinicopathologic phenotype and better prognosis in colorectal adenocarcinoma. Ann Surg Oncol 16: 297-303, 2009
19. Koda M, Sulkowska M, Kanczuga-Koda L, Surmacz E and Sulkowski S: Overexpression of the obesity hormone leptin in human colorectal cancer. J Clin Pathol 60: 902-906, 2007.

20. Viengchareun S, Zennaro MC, Pascual-Le Tallec L and Lombes M: Brown adipocytes are novel sites of expression and regulation of adiponectin and resistin. FEBS Lett 532: 345-350, 2002.

21. Beltowski J: Adiponectin and resistin-new hormones of white adipose tissue. Med Sci Monit 9: RA55-RA61, 2003.

22. Liu M and Liu F: Regulation of adiponectin multimerization, signaling and function. Best Pract Res Clin Endocrinol Metab 28: 25-31, 2014.

23. Aleksandrova K, Jenab M, Bueno-de-Mesquita HB, Fedirko V, Kaaks R, Lukanova A, van Duijnhoven FJ, Jansen E, Rinaldi S, Romieu I, et al: Biomarker patterns of inflammatory and metabolic pathways are associated with risk of colorectal cancer: Results from the European prospective investigation into cancer and nutrition (EPIC). Eur J Epidemiol 29: 261-275, 2014.

24. Chandran M, Phillips SA, Ciaraldi T and Henry RR: Adiponectin: More than just another fat cell hormone? Diabetes Care 26 2442-2450, 2003

25. Giovannucci E and Michaud D: The role of obesity and related metabolic disturbances in cancers of the colon, prostate, and pancreas. Gastroenterology 132: 2208-2225, 2007.

26. Catalán V, Gómez-Ambrosi J, Rodríguez A, Ramírez B, Silva C, Rotellar F, Hernández-Lizoain JL, Baixauli J, Valentí V, Pardo F, et al: Up-regulation of the novel proinflammatory adipokines lipocalin-2, chitinase-3 like-1 and osteopontin as well as angiogenic-related factors in visceral adipose tissue of patients with colon cancer. J Nutr Biochem 22: 634-641, 2011.

27. Kemik O, Kemik AS, Begenik H, Erdur FM, Emre H, Sumer A, Purisa S, Tuzun S and Kotan C: The relationship among acute-phase responce proteins, cytokines, and hormones in various gastrointestinal cancer types patients with cachectic. Hum Exp Toxicol 31: 117-125, 2012.

28. Al Khaldi RM, Al Mulla F, Al Awadhi S, Kapila K and Mojiminiyi OA: Associations of single nucleotide polymorphisms in the adiponectin gene with adiponectin levels and cardio-metabolic risk factors in patients with cancer. Dis Markers 30: 197-212, 2011.

29. Zekri AR, Bakr YM, Ezzat MM, Zakaria MS and Elbaz TM: Circulating levels of adipocytokines as potential biomarkers for early detection of colorectal carcinoma in Egyptian patients. Asian Pacific J Cancer Prev 16: 6923-6928, 2015.

30. American Joint Committee on Cancer: Chapter 20-Colon and rectum. In: AJCC cancer staging manual. 8th edition. Springer, New York, NY, 2017.

31. Dukes CE: The classification of cancer of the rectum. Dis Colon Rectum 23, 605-611, 1980.

32. Tessitore L, Vizio B, Jenkins O, De Stefano I, Ritossa C, Argiles JM, Benedetto C and Mussa A: Leptin expression in colorectal and breast cancer patients. Int J Mol Med 5: 421-426, 2000.

33. Kumor A, Daniel P, Pietruczuk M and Małecka-Panas E: Serum leptin, adiponectin, and resistin concentration in colorectal adenoma and carcinoma (CC) patients. Int J Colorectal Dis 24 275-281, 2009.

34. Sălăgeanu A, Tucureanu C, Lerescu L, Caras I, Pitica R, Gangurà G, Costea R and Neagu S: Serum levels of adipokines resistin and leptin in patients with colon cancer. J Med Life 3: 416-420, 2010

35. Ho GYF, Wang T, Gunter MJ, Strickler HD, Cushman M, Kaplan RC, Wassertheil-Smoller S, Xue X, Rajpathak SN, Chlebowski RT, et al: Adipokines linking obesity with colorectal cancer risk in postmenopausal women. Cancer Res 72: 3029-3037, 2012.

36. Kim HR: Obesity-related colorectal cancer: The role of leptin. Ann Coloproctol 31: 209-210, 2015.

37. Hellström L, Wahrenberg H, Hruska K, Reynisdottir S and Arner P: Mechanisms behind gender differences in circulating leptin levels. J Intern Med 247: 457-462, 2000.

38. Saad MF, Damani S, Gingerich RL, Riad-Gabriel MG, Khan A, Boyadjian R, Jinagouda SD, el-Tawil K, Rude RK and Kamdar V: Sexual dimorphism in plasma leptin concentration. J Clin Endocrinol Metab 82: 579-584, 1997.

39. Licinio J, Negrão AB, Mantzoros C, Kaklamani V, Wong ML, Bongiorno PB, Negro PP, Mulla A, Veldhuis JD, Cearnal L, et al: Sex differences in circulating human leptin pulse amplitude: Clinical implications. J Clin Endocrinol Metab 83: 4140-4147, 1998. 
40. Lu W, Huang Z, Li N and Liu H: Low circulating total adiponectin especially its non-high-molecular weight fraction, represents a promising risk factor for colorectal cancer: A meta-analysis. Onco Targets Ther 11: 2519-2531, 2018.

41. Rossi S, Basso M, Strippoli A, Schinzari G, D'Argento E, Larocca M, Cassano A and Barone C: Are markers of systemic inflammation good prognostic indicators in colorectal cancer? Clin Colorectal Cancer 16: 264-274, 2017.

42. Słomian G, Świętochowska E, Nowak G, Pawlas K, Żelazko A and Nowak P: Chemotherapy and plasma adipokines level in patients with colorectal cancer. Postepy Hig Med Dosw (Online) 71: 281-290, 2017.

43. Seker MM, Sancakdar E, Nadir A, Altun G, Bahceci A, Kacan T, Babacan NA and Kaptanoglu M: Serum leptin and adiponectin levels and relationship with prognosis in lung cancer. J Clin Oncol 32 (15 Suppl): e19114, 2014.

44. Moschovi M, Trimis G, Vounatsou M, Katsibardi K, Margeli A, Damianos A, Chrousos G and Papassotiriou I: Serial plasma concentrations of adiponectin, leptin, and resistin during therapy in children with acute lymphoblastic leukemia. J Pediatr Hematol Oncol 32: e8-e13, 2010.
45. Ayoub N, Alkhatatbeh M, Jibreel M and Ababneh M: Analysis of circulating adipokines in patients newly diagnosed with solid cancer: Associations with measures of adiposity and tumor characteristics. Oncol Lett 13: 1974-1982, 2017.

46. Carvajal-Carmona LG, Spain S; CORGI Consortium, Kerr D, Houlston R, Cazier JB and Tomlinson I: Common variation at the adiponectin locus is not associated with colorectal cancer risk in the UK. Hum Mol Genet 18: 1889-1892, 2009.

47. Partida-Pérez M, de la Luz Ayala-Madrigal M, Peregrina-Sandoval J, Macías-Gómez N, Moreno-Ortiz J, Leal-Ugarte E, Cárdenas-Meza M, Centeno-Flores M, Maciel-Gutiérrez V, Cabrales E, et al: Association of LEP and ADIPOQ common variants with colorectal cancer in Mexican patients. Cancer Biomark 7: 117-121, 2010.

48. Slattery ML, Wolff RK, Herrick J, Caan BJ and Potter JD: Leptin and leptin receptor genotypes and colon cancer: Gene-gene and gene-lifestyle interactions. Int J Cancer 122: 1611-1617, 2008.

49. Yang X, Li J, Cai W, Yang Q, Lu Z, Yu J, Yu H, Zhang N, Sun D, $\mathrm{Qu} \mathrm{Y}$, et al: Adiponectin gene polymorphisms are associated with increased risk of colorectal cancer. Med Sci Monit 21: 2595-606, 2015. 